\title{
DNA methylation of trefoil factor 1 (TFF1) is associated with the tumorigenesis of gastric carcinoma
}

\author{
GUOXUN FENG $^{1}$, YAN ZHANG ${ }^{2}$, HUISHENG YUAN $^{1}$, RIXING BAI ${ }^{1}$, \\ JIANWEI ZHENG ${ }^{1}$, JINQIAN ZHANG ${ }^{3}$ and MAOMIN SONG ${ }^{1}$
}

\author{
${ }^{1}$ Department of General Surgery, Beijing Tiantan Hospital, Capital Medical University, Beijing 100050; \\ ${ }^{2}$ Department of Ophthalmology, General Hospital of Beijing Military Area Command of Chinese PLA, \\ Beijing 100700; ${ }^{3}$ Institute of Infectious Diseases, Beijing Ditan Hospital, Capital Medical University, \\ Beijing Key Laboratory of Emerging Infectious Diseases, Beijing 100015, P.R. China
}

Received April 23,2013; Accepted September 5, 2013

DOI: $10.3892 / \mathrm{mmr} .2013 .1772$

\begin{abstract}
Trefoil factor 1 (TFF1) is a tumor suppressor gene that encodes a peptide belonging to the trefoil factor family of protease-resistant peptides. Although TFF1 expression is frequently lost in gastric carcinomas (GCs), the tumorigenic pathways that are affected have yet to be determined. The aim of the current study was to identify the mechanism(s) by which the TFF1 gene is regulated in gastric carcinogenesis. In this study, TFF1 was shown to be silenced or downregulated in gastric tumor tissue compared with matched non-cancerous tissue. In addition, human gastric cells weakly expressed TFF1. The hypermethylation status in the promoter $\mathrm{CpG}$ islands appeared to be correlated with TFF1 expression levels in gastric cell lines or specimen tissue. Further molecular analysis indicated that the $\mathrm{CpG}$ islands play a role in the promoter activity of the TFF1 gene. The expression of TFF1 and DNA methylation of its promoter affected cell proliferation and apoptosis. The expression of TFF1 in gastric cell lines was restored with a demethylating agent, 5-azacytidine. Low expression of TFF1 in gastric cell lines and cancer tissue is associated with TP 53. In conclusion, the current study demonstrates that DNA methylation is a key mechanism of silencing TFF1 expression in human gastric cells and TFF1 gene hypermethylation of the $\mathrm{CpG}$ islands is a potential biomarker for GC.
\end{abstract}

\section{Introduction}

Gastric cancer is one of the most common type of malignancies in China, of which the 5-year survival rate is $\leq 30 \%(1,2)$. The most common treatments include surgery and chemotherapy,

Correspondence to: Dr Maomin Song, Department of General Surgery, Beijing Tiantan Hospital, Capital Medical University, 6 Tiantan Xili, Dongcheng, Beijing 100050, P.R. China

E-mail: jingwanghou@yahoo.com.cn

Key words: DNA methylation, trefoil factor 1, tumorigenesis, gastric carcinoma however, these treatments are often accompanied with unsatisfactory effects. Therefore, there is a requirement for further study of its occurrence and the development of mechanisms to guide the treatment.

In the last three decades, cancer has been understood as a summary of altered genetic and epigenetic events. The epigenetic pathway is, in contrast to genetic events, a reversible alteration and is characterized by three primary mechanisms: i) DNA hypermethylation leading to inactivation; ii) DNA hypomethylation causing genomic instability and iii) histone modifications affecting chromatin conformation (3). These processes, particularly aberrant DNA methylation and histone modifications, are closely linked with each other by a protein complex of transcript activators and repressors, and they alter mRNA transcript expression of affected genes (4). Characteristically, DNA methylation does not change the genetic information. However, the readability of the DNA is altered and results in the inactivation of genes by subsequent mRNA transcript repression (3). In humans and other mammals, $\mathrm{CpG}$ island methylation is an important physiological mechanism. The inactivated $\mathrm{X}$-chromosome of female silenced alleles of imprinted genes or inserted viral genes and repeat elements are inactivated through promoter methylation $(5,6)$.

Moreover, with the progression of molecular biology in cancer, increasing evidence has indicated that the occurrence of gastric cancer results from multiple genetic alterations, including activated oncogenes, inactivation of tumor suppressor genes, loss of heterozygosity $(\mathrm{LOH})$ and defects in DNA damage response and repair mechanisms (3). Another factor which plays a key role in the regulation of gene expression is epigenetic changes in gene expression patterns due to mechanisms other than mutations in the underlying DNA sequence, including DNA methylation and histone modifications. According to a large number of animal tests, trefoil factor 1 (TFF1) plays a regulatory function in the mammals' digestive system, namely in mucosal protection and epithelial cell reconstruction, tumor suppression, signal transduction and the regulation of apoptosis.

The trefoil peptides (TFF1, TFF2 and TFF3) are a group of highly conserved small proteins, which are localized within the 
mucous granules in mucus-secreting cells and are expressed and secreted by epithelial cells which line mucous membranes (7). TFF1 is expressed predominantly by the gastric epithelia, in the upper portion of the glandular pits and is ectopically expressed in a number of adenocarcinomas, including breast cancer (7-9). In the breast, TFF1 expression is highly expressed in estrogen receptor-positive tumors and is inversely associated with histological grade (9). In the stomach, TFF1 is secreted as a component of the protective mucus layer. TFF1 is synthesized and secreted by the mucus-secreting pit cells of the corpus and antropyloric regions of the stomach $(7,10)$. TFF1 expression is markedly induced following mucosal injury (11) and is involved in stomach ontogenesis and maintenance of the integrity of the mucosa (7,8). Molecular studies have shown frequent loss of TFF1 expression in $>2 / 3$ of gastric carcinomas (GCs) resulting from a mutation-independent mechanism (12-14). The silencing of the TFF1 gene in GCs is due to LOH and methylation of the TFF1 promoter region $(12,15-18)$; however, mutations are observed in $\sim 5 \%$ of GCs $(12,19)$. Silencing of TFF1 may also be triggered by chromatin remodeling associated with histone modifications, including $\mathrm{H} 3 \mathrm{~K} 9$ methylation and $\mathrm{H} 3$ deacetylation at the TFF1 promoter, as observed in N-methyl-N-nitrosourea-induced gastric carcinogenesis mouse model (18). In addition, transcriptional repression of TFF1 in gastric epithelial cells by CCAAT/enhancer binding protein- $\beta$ (20) and cofactor of BRCA1 has been demonstrated (14). A previous study showed that TFF1 is a candidate tumor suppressor gene which inhibits cell growth (21). The TFF1-knockout mouse model provided the first evidence supporting a tumor suppressor role of TFF1 in gastric tumorigenesis, demonstrating that it is essential for normal differentiation of the antral and pyloric gastric mucosa (22). The spectrum of histological lesions and the mechanisms and molecular pathways, which are mediated by the loss of TFF1 in gastric tumorigenesis are not fully understood.

To determine the correlation between TFF1 and gastric cancer, the expression levels of TFF1 in GC tissue and cell lines was examined. To clarify the potential effect of DNA methylation in the promoter region in gastric carcinogenesis, the methylation rate and mechanism in specimens and cell lines was investigated. This study provides an experimental basis for gastric cancer diagnosis and clinical treatment, and provides a significant insight into epigenetic regulation in gastric cancer associated with TFF1.

\section{Materials and methods}

Cell culture and 5-aza-2'-deoxycytidine treatment. Human gastric cancer cell lines SGC-7901 and BGC-823 were cultured in Dulbecco's modified Eagle's medium supplemented with $10 \%$ fetal bovine serum and $1 \%$ antibiotics (penicillin-streptomycin solution) under identical conditions $\left(37^{\circ} \mathrm{C}\right.$ in humidified $5 \% \mathrm{CO}_{2} / 95 \%$ air). For drug treatment, cell lines were treated with $5 \mu \mathrm{mol} / 1$ 5-aza-2'-deoxycytidine (Aza; Sigma-Aldrich, St. Louis, MO, USA) for 3 days. Aza and medium were changed every $24 \mathrm{~h}$. Control cells were incubated with culture medium.

Tissue and surgical specimens. GC, adjacent non-tumor tissue and distant normal tissue specimens were obtained from 44 samples in the People's Liberation Army Air Force General
Hospital and People's Liberation Army General Hospital (Beijing, China) under Institutional Review Board-approved instructions. A further 15 normal tissue specimens adjacent to benign gastric ulcers were obtained from patients in the same two hospitals. The adjacent non-tumor tissues were removed $2 \mathrm{~cm}$ from the carcinoma border. Each non-tumor sample was divided into two groups and the distal section was used following confirmation that the proximal section was not cancerous. The normal tissue distant from carcinoma and those adjacent to benign gastric ulcer were removed $>5 \mathrm{~cm}$ from the carcinoma border or the ulcer. The samples were resected from surgical patients with pathological diagnosis between October 2002 and December 2012, prior to receiving any radiotherapy or chemotherapy. Following resection, all specimens were snap frozen in liquid nitrogen and stored at $-70^{\circ} \mathrm{C}$. All patient's age, gender and pathological type were recorded.

The use of human specimens in this research was approved by the Ethics Committee of the People's Liberation Army Air Force General Hospital and People's Liberation Army General Hospital (Beijing, China) according to the Declaration of Helsinki. All necessary consent was acquired from patients/patient's families involved in the study, including consent to participate in the study and consent to publish.

RNA extraction and reverse transcription-polymerase chain reaction $(R T-P C R)$. RNA was extracted from cells or patient tissue using an RNA isolation reagent (TRIzol; Invitrogen Life Technologies, Carlsbad, CA, USA). To prevent DNA contamination, total RNA was treated with RNase-free DNase II (Invitrogen Life Technologies).

The human glyceraldehyde-3-phosphate dehydrogenase gene (GAPDH; forward primer, 5'-TCACCAGGGCTGCTT TTA-3' and reverse primer, 5'-TTCACACCC ATGACGAACA-3') was used as an internal control in PCR amplification. A two-step RT-PCR procedure was performed in all experiments. First, total RNA samples ( $2 \mu \mathrm{g} /$ reaction $)$ were reverse transcribed into cDNA by RT II reverse transcriptase (Invitrogen Life Technologies). Then, the cDNAs were used as templates in PCR with TFF1 gene-specific primers (5'-TTTGGAGCAGAGAGGAGG-3' and 5'-TTGAGTAGTCAAAGTCAGAGCAG-3'). The primers for TP 53 were 5'-GGGTTAGTTTACAATCAGCCACATT-3' and 5'-GGGCCTTGAAGTTAGAGAAAATTCA-3'. The amplification reactions were performed using AmpliTaq Gold ${ }^{\circledR}$ DNA polymerase (Applied Biosystems, Foster City, CA, USA). The PCR cycle was programmed as follows: 2 min at $95^{\circ} \mathrm{C}$ and $30-32$ cycles of $30 \mathrm{sec}$ at $94^{\circ} \mathrm{C}, 30 \mathrm{sec}$ at $58-62^{\circ} \mathrm{C}$ and $30 \mathrm{sec}$ at $72^{\circ} \mathrm{C}$, with an extension for $10 \mathrm{~min}$ at $72^{\circ} \mathrm{C}$. The PCR bands were visualized under UV light and images were captured. Quantitative PCR was used to determine mRNA levels of urea cycle genes for hepatocellular carcinoma (HCC) cell lines and NOLC 1 mRNA levels for tissue on a StepOne Plus ${ }^{\mathrm{TM}}$ Real-Time PCR system with SYBR-Green Master Mix (Applied Biosystems).

Immunohistochemical staining. Biopsy specimens were subjected to routine immunohistochemical staining using a monoclonal antibody against TFF1. Immunoreactivity, defined as the number of positive tumor cells over total tumor cells, 
was scored independently by two researchers. The number of TFF1-positive and -negative gastric cancer cells was counted by light microscopy at a magnification of $x 400$, with only the cells exhibiting brown nucleoli on the section considered TFF1 positive. For each slide, 7-10 microscopic fields were randomly selected. Positive scores were then categorized into weak staining (only one nucleolus was stained), moderate staining ( $>1$ nucleolus was stained) and strong staining (the nucleus and nucleolus of the tumor cell staining). The average percentage of TFF1-positive gastric cancer cells was calculated for each group.

Western blot analysis. Lysates from the cultured cells were subjected to routine western blot analysis as described previously (23). The antibodies used were monoclonal anti-mouse antibodies against TFF1 and $\beta$-actin (Santa Cruz Biotechnology, Inc., Santa Cruz, CA, USA) and polyclonal rabbit antibodies against $\mathrm{NF}-\kappa \mathrm{B}$ and HIF-1 $\alpha$ (Abcam, Cambridge, MA, USA). The results shown are representative of two independent experiments.

Bisulfite genomic sequencing. Genomic DNA was purified from cells with a kit (Wizard Genomic DNA Purification kit; Promega Corporation, Madison, WI, USA). DNA (2 $\mu \mathrm{g})$ was bisulfite modified with the EZ DNA Methylation Direct kit; Zymo Research, Irvine, CA, USA). Sequence-specific primers to amplify the $\mathrm{CpG}$-rich regions of interest were designed using a computer program (MethPrimer; http://www.urogene. org/methprimer). The primers used for amplification were as follows: TFF1, 5'-TAGACGGAATGGGCTTCATG-3' (forward) and 5'-GCAAACAGAGCCTGCCCTAT-3' (reverse). The PCR products were amplified, purified and cloned into a vector (pGEM-T Easy Vector; Promega Corporation). Clones were selected using blue-white screening. Finally, the colonies harboring the insert were sequenced in a 96-well plate using the M13 reverse and/or forward primers.

The bisulfite-treated DNA was amplified using primers, which specifically amplify the methylated or unmethylated sequence of TFF1 promoter, respectively. The PCR was performed for 40 cycles, with an annealing temperature of $58^{\circ} \mathrm{C}$ for the methylated reaction and $52^{\circ} \mathrm{C}$ for the unmethylated reaction. The human methylated and unmethylated DNA was used as a control to verify the specificity of the primers (Qiagen, Valencia, CA, USA).

DNA samples from each group were cleaved by methylation-sensitive restriction enzyme or non-sensitive restriction enzyme, and this was followed by PCR amplification with the primer designed to be the DNA sequence outside where methylation was to be detected. Methylation in the relevant restriction enzyme site was confirmed if methylation was detected in the PCR products from the samples which used methylation-sensitive restriction enzyme. If no methylation was detected, then there was no methylation in the restriction enzyme site.

Plasmid and transfection. Cells were subcultured and transfected as previously described (24). The cDNA encoding TFF1, flanked by BamHI and SalI restriction sites, was cloned into the mammalian expression vector pCDNA4 (Stratagene Inc., La Jolla, CA, USA) to generate pCDNA-TFF1, which expresses an N-terminal Myc-tagged TFF1 fusion protein.
The promoter region of TFF1 was cloned into the pGL3 plasmid. Subconfluent cells were transiently transfected with pCDNA-TFF1 DNA (4 $\mu \mathrm{g} / \mathrm{dish})$ mixed with Lipofectamine and PLUS reagent (Invitrogen Life Technologies), according to the manufacturer's instructions. Cells were harvested $\sim 48 \mathrm{~h}$ following transfection (24).

RNA interference of TFF1. Stealth RNAi ${ }^{\mathrm{TM}}$ (Invitrogen Life Technologies) oligonucleotides for TFF1 were designed to target TFF1 mRNA and annealed as follows: siTFF1, 5'-AACAUGCAGUAA GGAUUCCACUUCC-3' (sense) and 5'-GGAAGUGGAA UCCUUACUGCAUGUU-3' (antisense); scrambled siRNA (negative control siRNA duplexes, 12935-300; Invitrogen Life Technologies). For transfection of siRNA, cells were plated into 6-well plates, grown until reaching $70-80 \%$ confluency and transfected with 40 or 80 pmol of siRNA duplex using Lipofectamine 2000 (Invitrogen Life Technologies) following the manufacturer's instructions.

Luciferase assay. The cells were transfected with $0.6 \mu \mathrm{g}$ of firefly luciferase reporter plasmid and $0.05 \mu \mathrm{g}$ of control plasmid containing Renilla luciferase (pRL-TK; Promega Corporation). A promoterless basic vector (pGL3; Promega Corporation) was used as a negative control. To confirm the efficiency of transfection (Lipofectin; Invitrogen Life Technologies), a luciferase expression vector (pGL3-control; Promega Corporation) was used as a positive control. Following $48 \mathrm{~h}$, the cells were harvested for analysis. Luciferase enzyme assays and colorimetric $\beta$-galactosidase assays were performed according to the manufacturer's instructions (Promega Corporation). Luciferase activity was normalized to $\beta$-galactosidase activity to assess the transfection efficiency. When indicated, the firefly and Renilla luciferase activities were measured using the Dual-Luciferase Reporter Assay system (Promega Corporation), according to the manufacturer's instructions. The Renilla luciferase activity of pRL-TK was used to normalize the firefly luciferase activity of the reporter construct. Each transfection experiment was repeated three times.

Cell growth and proliferation assay. Cell growth was determined by the colorimetric tetrazolium derived sodium 3'-[1-(phenylamino-carbonyl)-3,4-tetrazolium]-bis(4-methoxy -6-nitro) benzenesulfonic acid hydrate (XTT) assay (Roche Diagnostics GmbH, Mannheim, Germany) and DNA synthesis of cells was assessed by the bromodeoxyuridine (BrdU) incorporation assay (Roche Diagnostics $\mathrm{GmbH}$ ). For the cell growth and proliferation assay, the cells of each group at $48 \mathrm{~h}$ following treatment were re-seeded onto 96-well plates at a density of $3 \times 10^{3}$ cells/well. Then, XTT and incorporated BrdU were measured colorimetrically using a microtiter plate reader (Bio-Rad, Hercules, CA, USA) at a wavelength of $450 \mathrm{~nm}$ (25).

Determimation of apoptosis. Cells $\left(3 \times 10^{5}\right)$ were cultured for $48 \mathrm{~h}$. Apoptotic cells were identified using fluorescence-activated cell sorting (FACS) Annexin V-FLUOS (BioLegend, San Diego, CA, USA) following the manufacturer's instructions. Following culturing, cells were washed at $4^{\circ} \mathrm{C}$ for $30 \mathrm{~min}$ in PBS and stained with Annexin-V staining solution (Annexin-V-FLUOS kit; Roche Diagnostics $\mathrm{GmbH}$ ) at $4^{\circ} \mathrm{C}$ 
$\mathbf{A}$

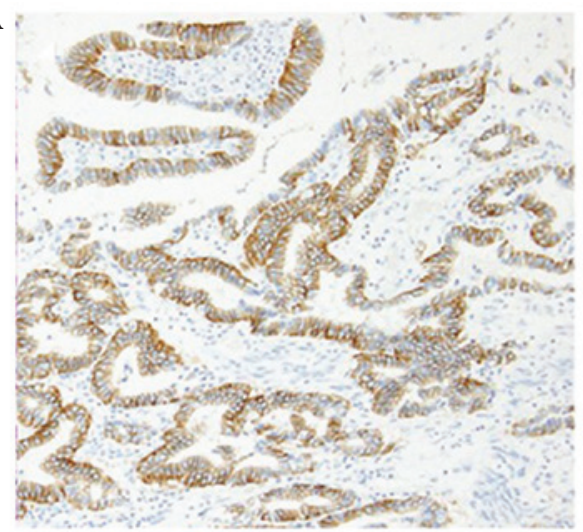

C

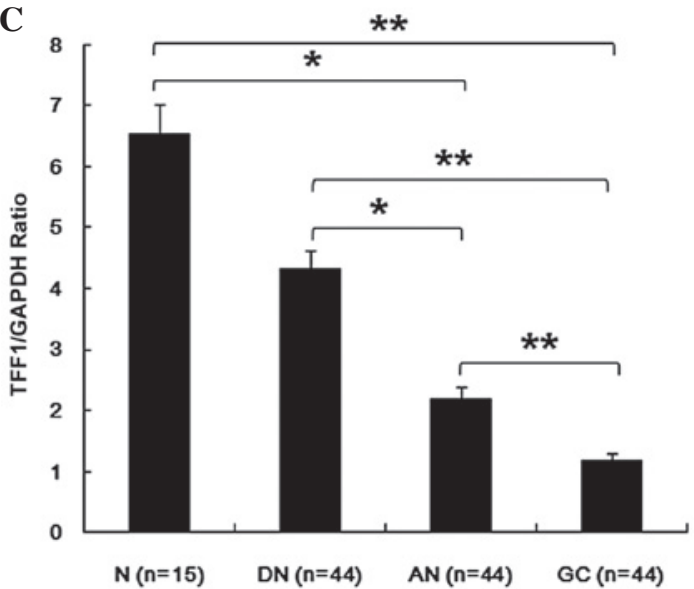

B

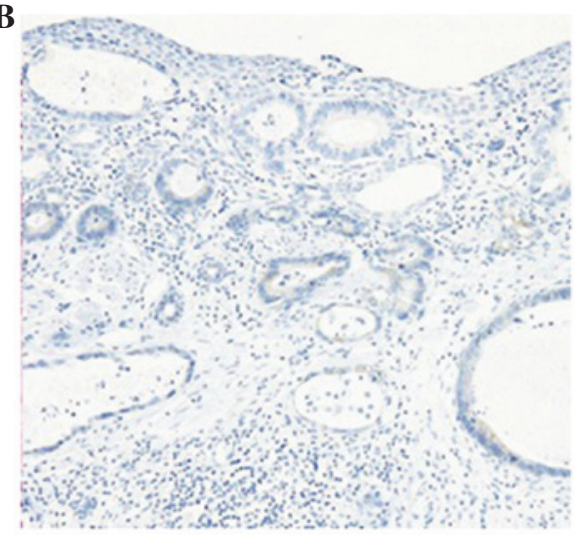

D

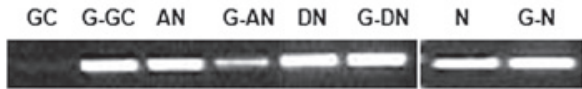

$\mathbf{E}$

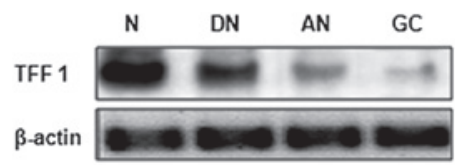

Figure 1. TFF1 mRNA and protein expression in gastric cancer cell lines and patient specimens. (A) The protein expression of TFF1 in normal tissue adjacent to $\mathrm{N}$ by IH staining. (B) The protein expression of TFF1 in tumor tissues of patients with GC by IH and DN, AN and GC. (C) TFF1 mRNA levels in tissues were detected by RT-PCR in patients with gastric ulcer $(\mathrm{n}=15)$ and patients with gastric carcinoma $(\mathrm{n}=44 ; \mathrm{N}$ vs. AN, $\mathrm{P}=0.032 ; \mathrm{N}$ vs. $\mathrm{GC}, \mathrm{P}=0.000 ; \mathrm{DN}$ vs. $\mathrm{AN}$, $\mathrm{P}=0.018$; DN vs. GC, $\mathrm{P}=0.004$; AN vs. GC, $\mathrm{P}=0.000$ ). (D) TFF1 mRNA levels in tissues were detected by quantitative PCR in patients with gastric ulcer ( $\mathrm{n}=15$ ) and patients with gastric carcinoma $(\mathrm{n}=44)$. (E) TFF1 protein levels in tissues were detected by western blot analysis in patients with gastric ulcer or GC. The data are presented as the mean $\pm \mathrm{SD}$ from three independent experiments ${ }^{*} \mathrm{P}<0.05,{ }^{* *} \mathrm{P}<0.01$. TFF1, trefoil factor 1 ; IH, immunohistochemical; RT-PCR, reverse transcription-polymerase chain reaction; GAPDH, glyceraldehyde-3-phosphate dehydrogenase; N, benign gastric ulcer group; DN, distant normal tissue of patients with gastric carcinoma; AN, adjacent non-tumor of patients with gastric carcinoma; GC, tumor tissues of patients with gastric carcinoma.

for 3 h. Gels were washed 4 times in PBS at $4^{\circ} \mathrm{C}$ and fixed at room temperature with $1 \%$ paraformaldehyde (Sigma-Aldrich) in PBS for $15 \mathrm{~min}$. For counterstaining, 7-AAD $(2 \mu \mathrm{g} / \mathrm{ml})$ was added to the first washing step. The numbers of total, Annexin-V-positive, 7-AAD-positive and double-positive cells were determined respectively by FACS analysis. Apoptosis was verified by detection of activated caspases (26).

Statistical analysis. Using GraphPad Prism software, a 2-tailed Student's t-test was used to compare the statistical difference between 2 groups and a one-way ANOVA Newman-Keuls Multiple Comparisons test was used to compare the differences between 3 groups or more. The correlation between 2 parameters, age and chronic inflammation scores, was determined by Spearman's correlation. $\mathrm{P} \leq 0.05$ was considered to indicate a statistically significant difference.

\section{Results}

Low expression of TFF1 in gastric cancer tissue is associated with TP 53. To determine the potential mechanisms by which TFF1 is regulated in GC, TFF1 expression levels at the transcription and translation level in the tissue of patients was determined ( $\mathrm{N}$, normal tissues adjacent to benign gastric ulcer; $\mathrm{DN}$, distant normal tissue of patients with GC; $\mathrm{AN}$, adjacent non-tumor of patients with GC; GC, GC tissue of patients with GC) using immunohistochemical staining and RT-PCR. The results show that TFF1 was moderately expressed in the mucosa of normal tissue adjacent to benign gastric ulcer (Fig. 1A) and was expressed at a low level in GC tissue (Fig. 1B). The expression levels of TFF1 mRNA was examined in 44 specimens of GC, adjacent non-tumor, distant normal tissues and 15 cases of normal tissue from patients with benign gastric ulcer, using RT-PCR. The expression levels in GC specimens were significantly lower compared with the other three specimens $(\mathrm{P}<0.01)$. The results in adjacent non-tumor tissue were significantly lower compared with the distant normal tissue and the normal tissues adjacent to a benign gastric ulcer $(\mathrm{P}<0.05)$. By contrast, the expression level of TFF1 between the adjacent non-tumor and normal tissue adjacent to benign gastric ulcer showed no significant difference ( $P>0.05$; Fig. $1 C$ and D). To determine the association of mRNA transcription with protein expression, western blot analysis was performed to examine the TFF1 protein expression in patient tissues. As shown in 


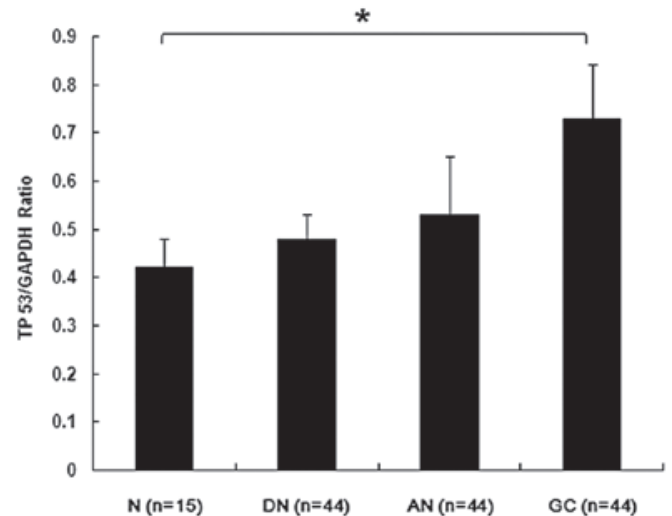

Figure 2. TP 53 mRNA expression levels in the tissue of patients. The TP 53 mRNA expression levels were analyzed by RT-PCR in normal and tumor tissues of patients with HCC. GAPDH was used as an internal control of the RT-PCR for normalization. The data are presented as the mean $\pm \mathrm{SD}$. ${ }^{*} \mathrm{P}<0.05$, vs. control (N vs. GC, $\mathrm{P}=0.014$ ). RT-PCR, reverse transcription-polymerase chain reaction; HCC, hepatocellular carcinoma; GAPDH, glyceraldehyde-3-phosphate dehydrogenase; N, normal tissues adjacent to benign gastric ulcer; DN, distant normal tissue of patients with gastric carcinoma; AN, adjacent non-tumor of patients with gastric carcinoma; GC, gastric carcinoma tissue of patients with gastric carcinoma.

Fig. 1E, a strong expression of TFF1 protein was detected in normal tissue adjacent to benign gastric ulcer and weakly expressed in distant normal tissue of patients with GC. TFF1 protein decreased significantly in adjacent noncancerous specimens, particularly in the tumor samples of patients.

Fenoglio-Preisern et al (27) found that p53 alterations occur early in the development of GC and are present in the non-neoplastic mucosa and increase in frequency as GC development progresses. p53 immunoreactivity is observed in $17-90.7 \%$ of invasive GCs. p53 alterations occur more commonly in proximal lesions compared with distal ones, suggesting that the molecular events leading to the development of GC may be markedly different in proximal vs. distal tumors. p53 mutations occur in $0-77 \%$ of GCs (27). TP 53 mRNA expression was then examined in patient tissue. The TP 53 expression in tumor tissue was higher compared with normal tissue adjacent to benign gastric ulcer (Fig. 2).

TFF1 expression is regulated by DNA methylation in GC tumor cells. Since numerous cancer cells exhibit aberrant epigenetic regulation, it is possible that TFF1 expression is regulated by epigenetic modification. To confirm that DNA methylation regulates TFF1 expression, detailed methylation analysis of the TFF1 gene sequence was performed using genomic DNA extracted from cell lines and gastric tissue. To determine whether TFF1 promoter methylation is relevant to TFF1 expression control in gastric cancer cell lines and clinical specimens, methylation-specific PCR was performed and compared with the promoter methylation status in gastric cell lines SGC-7901 and in tumor tissue with that in normal tissue adjacent to benign gastric ulcers. Methylation of the $\mathrm{CpG}$ dinucleotides in the promoter region was detectable. As shown in Fig. 3, the results revealed that TFF1 were markedly methylated in gastric cancer cell lines and tumor tissue (Fig. 3). The methylation status in the promoter region appears to be correlated with TFF1 expression levels in gastric cell lines or specimen tissue.

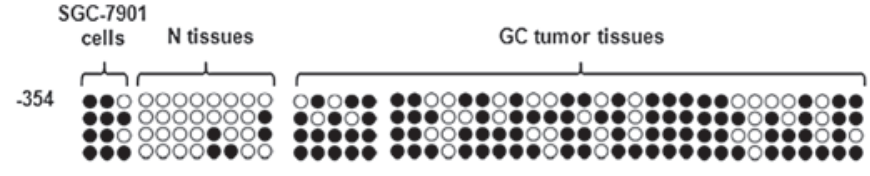

Figure 3. Regulation of TFF1 expression by DNA methylation in GC. The DNA methylation status of SGC-7901 cell lines and tissues of patients is shown. Methylated sites are indicated by filled dark circles and unmethylated sites by empty white circles. TFF1, trefoil factor $1 ; \mathrm{N}$, normal tissues adjacent to benign gastric ulcer; GC, gastric carcinoma tissues of patients with gastric carcinoma.
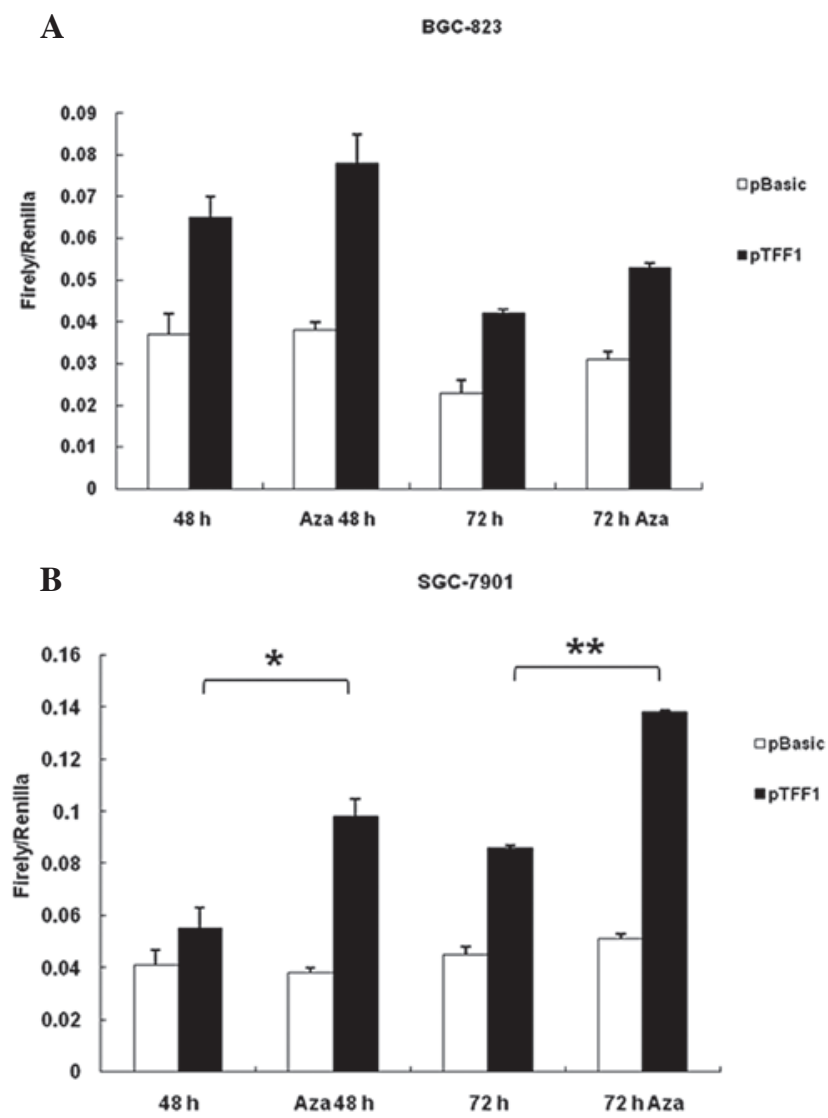

Figure 4. Luciferase reporter assay of TFF1 minimal promoter activity. (A) BGC-823 and (B) SGC-7901 cells were transfected with reporter gene plasmid (pGL3-TFF1), together with pRL-TK Renilla luciferase plasmid, as an internal control. Following $24 \mathrm{~h}$ transfection, the cells were treated with or without Aza for a subsequent 24 or $48 \mathrm{~h}$ ( $48 \mathrm{~h}$ vs. $48 \mathrm{~h}$ Aza, $\mathrm{P}=0.023$; $72 \mathrm{~h}$ vs. $72 \mathrm{~h} \mathrm{Aza}, \mathrm{P}=0.000$ ). The cells were harvested and luciferase activity was determined using a Dual Luciferase kit. The data are presented as the mean \pm SD from three independent experiments. ${ }^{*} \mathrm{P}<0.05,{ }^{* *} \mathrm{P}<0.01$. TFF1, trefoil factor 1; Aza, 5-aza-2'-deoxycytidine.

Effect of the CpG1 island on TFF1 promoter activity. To investigate the possible effect of methylation of the promoter activity and to determine the functional significance, a reporter gene construct was generated using a TFF1 promoter sequence containing $\mathrm{CpG}$ island. The reporter construct (pTFF1), together with pRL-TK Renilla luciferase expression vectorU, were transiently transfected in BGC-823 and SGC-7901 cells, followed by Aza treatment. The promoter activity was determined by luciferase assay. Firefly and Renilla luciferase activities were measured at the point indicated. Renilla luciferase activity was used to normalize 
A

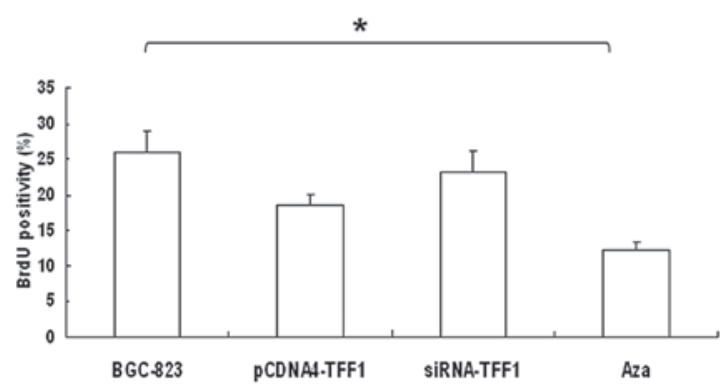

C

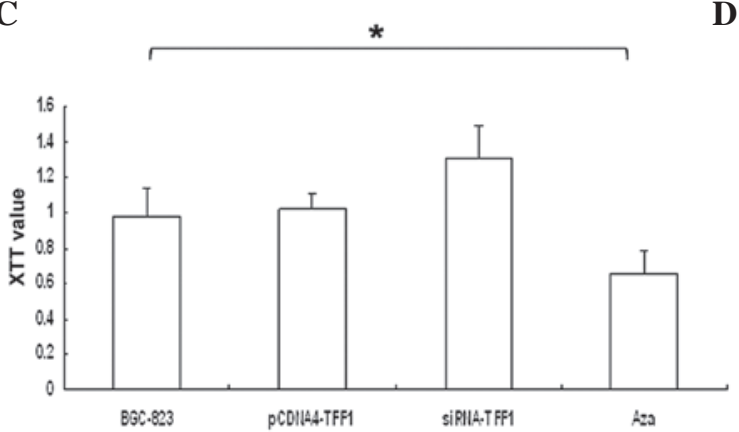

B

D
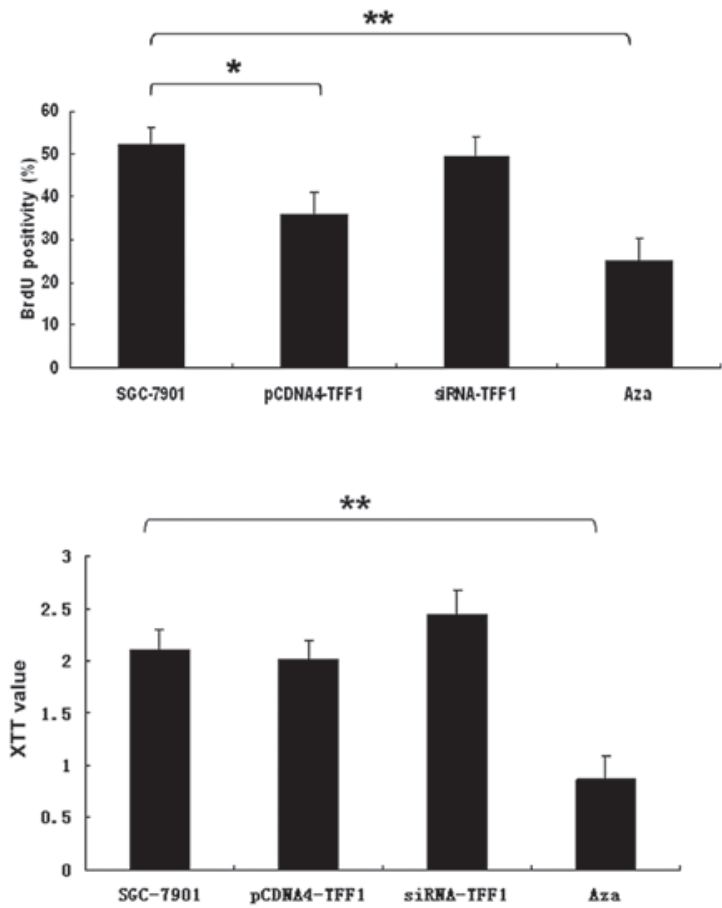

Figure 5. Expression of TFF1 and DNA methylation of its promotor affected cell proliferation. (A and C) BGC-823 and (B and D) SGC-7901 cells were transfected with plasmid pCDNA4-TFF1 or siRNA-TFF1. Following $24 \mathrm{~h}$ transfection, the cells were treated with or without Aza for a subsequent $24 \mathrm{~h}$. The effects of expression of TFF1 and DNA methylation of its promoter on cell growth and viability were determined by BrdU and XTT assay. All data are presented as the mean \pm SEM. (A) $\mathrm{P}=0.011$; (B) SGC7901 vs. Aza, $\mathrm{P}=0.000$; SGC7901 vs. pCDNA-TFF1, $\mathrm{P}=0.031$; (C) $\mathrm{P}=0.043$; (D) $\mathrm{P}=0.001 .{ }^{*} \mathrm{P}<0.05$, ** $\mathrm{P}<0.01$. TFF1, trefoil factor 1; Aza, 5-aza-2'-deoxycytidine; BrdU, bromodeoxyuridine; XTT, sodium 3'-[1-(phenylamino-carbonyl)-3,4-tetrazolium]-bis(4-methoxy-6-nitro) benzenesulfonic acid hydrate.

firefly luciferase activity of the reporter constructs. As shown in Fig. 4, Aza treatment caused a significant increase in promoter activity in the two cell lines, particularly in SGC-7901 cells (Fig. 4B). Luciferase activity of pGL3-basic, which has no promoter element, was not affected by Aza treatment. The $\mathrm{CpG}$ islands in the plasmid were not methylated at transfection (data not shown). Thus, the data suggest that the $\mathrm{CpG}$ islands appear to be critical for TFF1 promoter activity.

Function of TFF1 in cell biology. It is unclear how TFF1 affects cell function. Cells were synchronized at the G1/S boundary by double thymidine block and underwent mitosis. Following $24 \mathrm{~h}$, BrdU was added into the medium at indicated time points to evaluate DNA synthesis. As shown in Fig. 5A, incorporation of BrdU into the control, accumulation of mitotic BGC-823 cells was significantly inhibited by TFF1 siRNA $(\mathrm{P}<0.05)$. By contrast, overexpression of TFF1 in SGC-7901 cells were significantly delayed at $48 \mathrm{~h}(\mathrm{P}<0.05)$, particularly in cells treated with Aza $(\mathrm{P}<0.01)$ (Fig. 5B).

By XTT assays, TFF1 silencing of BGC-823 cells resulted in a significant decreased of cell growth when compared with control and others $(\mathrm{P}<0.05$; Fig. 5C). However, Aza treatment in SGC-7901 cells significantly inhibited cell growth compared with the control group $(\mathrm{P}<0.01$; Fig. 5D).

The effect of TFF1 expression on apoptosis distribution was determined in BGC-823 and SGC-7901 cells by flow cytometry. Downregulation of TFF1 in BGC-823 cells promoted significant apoptosis $(\mathrm{P}<0.01$; Fig. 6A). Furthermore,
SGC-7901 cells treated with Aza induced an increase in apoptosis $(\mathrm{P}<0.01$; Fig. 6B). This maybe associated with low levels of TP 53, particularly in SGC-7901 (Fig. 7B).

\section{Discussion}

It is well established that carcinogenesis undergoes multistep progression, which includes gastric mucosa, chronic gastritis, atrophy, intestinal metaplasia and dysplasia, with the involvement of multiple genetic alterations (28). The molecular changes of DNA are represented by the activation of oncogenes, inactivation of tumor suppressor genes, $\mathrm{LOH}$ and defects in DNA damage response and repair mechanisms. Another factor which plays a key role in the regulation of gene expression is epigenetic changes in gene expression patterns due to mechanisms other than mutation in the underlying DNA sequence, including DNA methylation and histone modifications (29).

The TFF1 gene has been localized to chromosome 21q, the long arm of human chromosome 21 and has been identified with three exons, two introns and two promoters. The small molecule peptide TFF1 is primarily secreted by gastrointestinal mucosa cells and has been confirmed to function in gastrointestinal mucosa protection and repair (30). Under normal conditions, the TFF1 gene is primarily expressed in mucosal epithelial cells in the gastric body and antrum, while under pathological conditions, its expression specificity disappears (8) and TFF1 may be secreted in any gastrointestinal mucosal injury sites (31). The molecular forms of TFF1 identified in normal human gastric mucosa are: TFF1 monomer, 
TFF1 dimer which is rather rare and the predominant TFF1 complex with a molecular mass of $\sim 25 \mathrm{kDa}$ (32). According to a large number of animal tests, trefoil peptide conducts regulatory functions on mammals, namely mucosal protection and epithelial cell reconstruction, tumor suppression, signal transduction and regulation of apoptosis (28).

In a previous study (30), an altered DNA methylation pattern was found to play a definitive role in the regulation of TFF1 gene expression in human gastric cancers. In the current study, there is evidence to support the hypothesis that DNA methylation is a key mechanism of epigenetic regulation to suppress TFF1 expression in gastric cancer cells and identify the precise methylation area in the TFF1 gene. This study provides an important insight into epigenetic regulation in GC.

Aberrant epigenetic states may predispose an individual to genetic changes; however, genetic changes may also initiate aberrant epigenetic events. Epigenetic and genetic mechanisms may thus work together to silence key cellular genes and destabilize the genome, leading to oncogenic transformation and observed the complexity and heterogeneity in human cancers, including GC (33-35). The development of GC results from a multistep process beginning with the accumulation of genetic and epigenetic alterations in regulatory genes (3). In general, cancer cells have global hypomethylation; however, they exhibit hypermethylation in specific genes. DNA hypermethylation in promoter regions is associated with silencing of tumor suppressor genes due to direct or indirect prevention to accessing transcription factors in the promoter region (36). Previous studies $(3,37,38)$ have demonstrated that $\mathrm{CpG}$ island hypermethylation, via silencing of key cancer-related genes, plays a major causal role in cancer, including GC.

The current study of TFF1 gene methylation provides a novel insight into the epigenetic regulation in GC. In the study, it is clear that TFF1 expression is suppressed in gastric cancer cell lines and tumor tissue. Moreover, TFF1 expression in gastric cancer cell lines may be restored by treatment with the demethylating agent Aza, particularly in high differentiation SGC-7901 associated with TP 53, but not associated with low differentiation BGC-823. We demonstrate further that the methylation status of the $\mathrm{CpG}$-rich area in the promoter region is correlated with TFF1 gene expression in gastric cancer cell lines. The enhanced TFF1 promoter activity in a reporter assay in the gastric cancer cell lines was tested. These observations indicate that DNA methylation regulates TFF1 expression in gastric cancer cells.

The majority of studies investigating the mechanisms which regulate gene expression by $\mathrm{CpG}$ methylation focus on $\mathrm{CpG}$ islands in the promoter. In the present study, the TFF1 promoter region (-354) was found. Hypermethylation of $\mathrm{CpG}$ islands in promoter sequences is associated with silencing of tumor suppressor genes and tumor-related genes by subsequent downregulation of mRNA transcription expression. Epigenetic silenced genes are involved in important molecular pathways of carcinogenesis e.g., cell cycle regulation, apoptosis, DNA repair or cell adhesion. The imbalance between cell proliferation and death is considered to be an early and significant event in the carcinogenic process, thus, it is desirable to develop a new strategy to induce apoptosis and proliferation inhibition in tumor cells. The results of the study demonstrated that TFF1 inhibits the proliferation of gastric cancer cell lines and

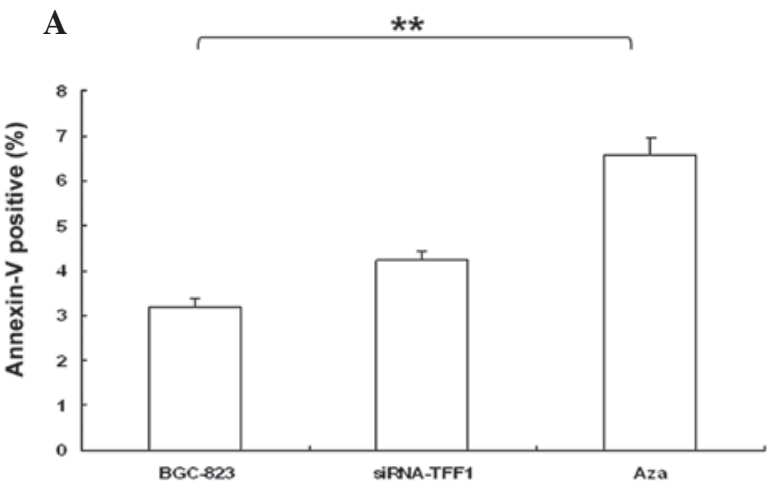

B

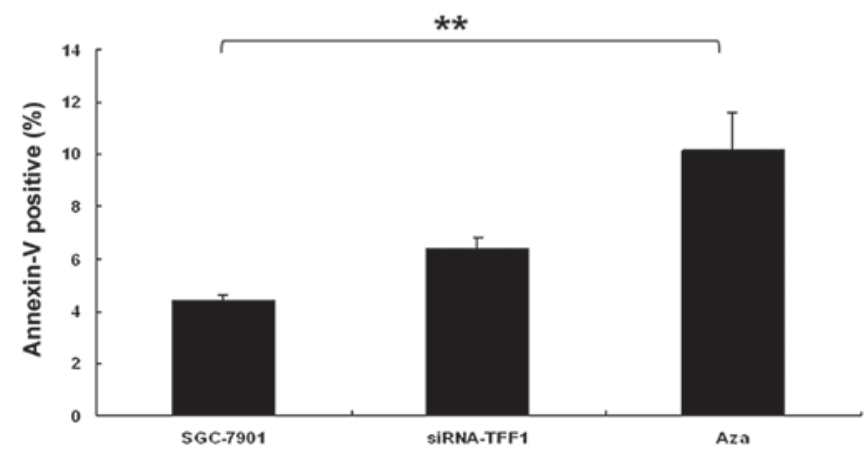

Figure 6. Expression of TFF1 and DNA methylation of its promotor affected cell apoptosis. (A) BGC-823 and (B) SGC-7901 cells were transfected with plasmid siRNA-TFF1. Following $24 \mathrm{~h}$ culture, the cells were treated with or without Aza for a subsequent $24 \mathrm{~h}$. The effects of expression of TFF1 and DNA methylation of its promoter on cell apoptosis were determined by FACS analysis using Annexin V-FLUOS (BioLegend) following the instructions of the manufacturer. All data are presented as the mean \pm SEM. (B) $P=001$ ${ }^{* * *} \mathrm{P}<0.01$. TFF1, trefoil factor 1; Aza, 5-aza-2'-deoxycytidine; FACS, fluorescence-activated cell sorting.

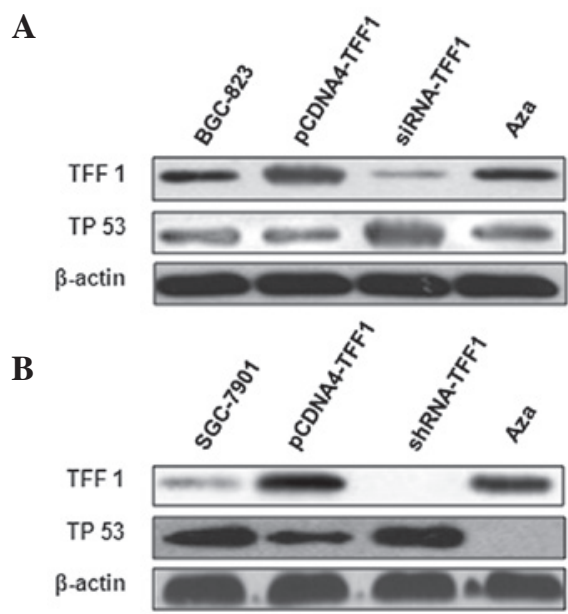

Figure 7. Functional characterization of TFF1 involves TP 53. (A) BGC-823 and (B) SGC-7901 cells were transfected with plasmid pCDNA4-TFF1 or siRNA-TFF1. Following $24 \mathrm{~h}$ transfection, the cells were treated with or without Aza for a subsequent $24 \mathrm{~h}$. The effects of expression of TFF1 and DNA methylation of its promoter on the expression of TP 53 were studied by western blot analysis. TFF1, trefoil factor 1; Aza, 5-aza-2'-deoxycytidine.

promotes apoptosis. TFF1 is hypothesized to play a role in suppressing GC tumorigenesis.

Another observation in this experiment was the high methylation rate in GC. Therefore, it is hypothesized that 
methylation may be involved in the downregulation of TFF1 expression in GC while the particular molecular mechanism remains to be defined. Methylation is a salient feature of mammalian genomes. It promotes gene diversity of humans, while the loss of DNA methylation may result in gene mutation and rearrangement, and contributes to genomic instability. In the human body, methylation only occurs on cytosine residues, primarily those in $\mathrm{CpG}$ islands. Under normal conditions, $\mathrm{CpG}$ islands are not methylated if they are located upstream of house-keeping genes, which is expressed at relatively constant levels. The high methylation levels of house-keeping genes may inhibit their expression. In addition, hypomethylation in the overall genome level and hypermethylation in a number of particular gene sites, particularly promoter sequences, is likely to break the normal methylation pattern and impact gene expression regulation and cellular differentiation (39).

A number of studies observed potential connections between hypermethylation in promoter sites and inactivation of genes related to specific familial cancers, including RBI, CHL, E-cad and P16. There are a number of genes in gastric cancer where the promoter methylation rate is $>50 \%$ : Cox22 (86.6\%) (40), E-cadherin (75.9\%), DAP-kinase (70.3\%), P15 (68.5\%), P16 (66.7\%) (41), 06-MGMT (61\%) (42), RASSFIA (60\%) (43), TMP-3 (57.4\%) (44). Furthermore, the positive rate in multigene methylation detection is significantly higher compared with the detections at a single gene. In short, promoter methylation in cancer-related genes is an early and frequent event during carcinogenesis. Therefore, it holds a biological and clinical significance in the early diagnosis for gastric cancer, which is essential to cancer treatment and follow-up of surgical patients. In addition, DNA methylation is beneficial for cancer diagnosis since it is not only detected in resected tissues, but also in various body fluids, including fluids in cancerous organs, peripheral blood, saliva and sputum (44). To conclude, promoter methylation in cancer-related genes is an extremely promising novel biomarker.

By contrast, DNA methylation is a reversible process and gene expression may be restored with methylation inhibitors (45). Thus, the normal growth regulation pattern may be restored by demethylating genes prior to mutation or other damages. It may also be a possible pathway for cancer treatment in the future.

The marked downregulation or absence of TFF1 mRNA and protein expression in GC tissue specimens and high expression level in adjacent non-tumor tissue suggests that TFF1 may be involved in the inhibition of gastric cancer. Adjacent non-tumor tissues secrete additional TFF1 to suppress tumor growth. Moreover, according to previous literature, the TFF1 expression level in the gastric mucosa which borders gastric ulcers is higher compared with the distant mucosa (46-49). The high expression level in mucosa adjacent to GC or gastric ulcer suggests that TFF1 plays a significant role in mucosal protection and epithelial cell reconstruction. A potential mechanism by which this occurs is by enhancing the replication of adjacent intact epithelial cells to replace the damaged mucous membrane. In addition, there are two possible causes for the low expression level or absence of TFF1 in GC: i) potential genetic alterations, including gene mutation, LOH and DNA methylation and ii) poorly differentiated glands and cells, which are too damaged to secrete TFF1. In conclusion, TFF1 is a potential suppressor of gastric cancer and may be involved in inhibiting gastric carcinogenesis and development.

According to the results of these experiments, the TFF1 promoter site was hypermethylated, the proportion being $40.91 \%$. The location of these methylated sites was among three CmCGG (cytosine-methylcytosine-guanine-guanine) sequences: $-354,-84$ and $-20 \mathrm{nt}$, particularly in -354 . Although in the other $\sim 60 \%$ carcinoma samples, no methylation was detected, the TFF1 mRNA and protein expression levels were significantly downregulated. There are two possible causes for this phenomenon: i) methylation in other sites which are outside the promoter region and ii) gene mutation, $\mathrm{LOH}$ or other genetic alterations. DNA methylation blocks the tumor suppressor role of the TFF1 gene since the secretion of TFF1 mRNA and peptides were affected.

The marked downregulation of TFF1 secretion in GC suggests that TFF1 may be an inhibitory factor of gastric cancer and that the TFF1 gene may be a tumor suppressor gene. Also, the high methylation rate in TFF1 of GC suggests that methylation in the TFF1 DNA promoter region may attribute to the reduced or absent TFF1 mRNA expression, hence carcinogenesis of gastric cancer. This study provides a potential target point for the future treatment of gastric cancer, however the molecular mechanisms for TFF1 in carcinogenesis and progression of gastric cancer remains to be studied further. There are a number of hypotheses which indicate that DNA methylation in the promoter region is reversible. All these studies are invaluable for further cancer research and biologically targeted therapy for cancer treatment, particularly in GC associated with TFF1.

\section{Acknowledgements}

This study was supported by grants from the National Natural Science Foundation of China (nos. 30600524, 81071990, 81172383 and 81201758).

\section{References}

1. Stadtländer CT and Waterbor JW: Molecular epidemiology, pathogenesis and prevention of gastric cancer. Carcinogenesis 20: 2195-2208, 1999.

2. Ren J, Chen Z, Juan SJ, Yong XY, Pan BR and Fan DM: Detection of circulating gastric carcinoma-associated antigen MG7-Ag in human sera using an established single determinant immuno-polymerase chain reaction technique. Cancer 88: 280-285, 2000.

3. Tischoff I and Tannapfel A: DNA methylation in hepatocellular carcinoma. World J Gastroenterol 14: 1741-1748, 2008.

4. Baylin SB and Herman JG: DNA hypermethylation in tumorigenesis: epigenetics joins genetics. Trends Genet 16: 168-174, 2000.

5. Riggs AD and Pfeifer GP: X-chromosome inactivation and cell memory. Trends Genet 8: 169-174, 1992.

6. Razin A and Cedar H: DNA methylation and genomic imprinting. Cell 77: 473-476, 1994.

7. Thim L and May FE: Structure of mammalian trefoil factors and functional insights. Cell Mol Life Sci 62: 2956-2973, 2005.

8. Ribieras S, Tomasetto C and Rio MC: The pS2/TFF1 trefoil factor, from basic research to clinical applications. Biochim Biophys Acta 1378: F61-F77, 1998.

9. Corte MD, Tamargo F, Alvarez A, Rodríguez JC, Vázquez J, Sánchez R, Lamelas ML, González LO, Allende MT, García-Muñiz JL, Fueyo A and Vizoso F: Cytosolic levels of TFF1/pS2 in breast cancer: their relationship with clinical-pathological parameters and their prognostic significance. Breast Cancer Res Treat 96: 63-72, 2006. 
10. Rio MC, Bellocq JP, Daniel JY, Tomasetto C, Lathe R, Chenard MP, Batzenschlager A and Chambon P: Breast cancer-associated pS2 protein: synthesis and secretion by normal stomach mucosa. Science 241: 705-708, 1988.

11. Taupin D, Pedersen J, Familari M, Cook G, Yeomans N and Giraud AS: Augmented intestinal trefoil factor (TFF3) and loss of pS2 (TFF1) expression precedes metaplastic differentiation of gastric epithelium. Lab Invest 81: 397-408, 2001.

12. Carvalho R, Kayademir T, Soares P, Canedo P, Sousa S, Oliveira C, Leistenschneider P, Seruca R, Gött P, Blin N, Carneiro F and Machado JC: Loss of heterozygosity and promoter methylation, but not mutation, may underlie loss of TFF1 in gastric carcinoma. Lab Invest 82: 1319-1326, 2002.

13. Katoh M: Trefoil factors and human gastric cancer (review). Int J Mol Med 12: 3-9, 2003.

14. McChesney PA, Aiyar SE, Lee OJ, Zaika A, Moskaluk C, Li R and El-Rifai W: Cofactor of BRCA1: a novel transcription factor regulator in upper gastrointestinal adenocarcinomas. Cancer Res 66: 1346-1353, 2006.

15. Park WS, Oh RR, Park JY, Yoo NJ, Lee SH, Shin MS, Kim SY, Kim YS, Lee JH, Kim HS, An WG and Lee JY: Mapping of a new target region of allelic loss at 21q22 in primary gastric cancers. Cancer Lett 159: 15-21, 2000.

16. Ribieras S, Lefèbvre O, Tomasetto $\mathrm{C}$ and Rio MC: Mouse Trefoil factor genes: genomic organization, sequences and methylation analyses. Gene 266: 67-75, 2001.

17. Fujimoto J, Yasui W, Tahara H, Tahara E, Kudo Y, Yokozaki H and Tahara E: DNA hypermethylation at the $\mathrm{pS} 2$ promoter region is associated with early stage of stomach carcinogenesis. Cancer Lett 149: 125-134, 2000.

18. Tomita H, Takaishi S, Menheniott TR, Yang X, Shibata W, Jin G, Betz KS, Kawakami K, Minamoto T, Tomasetto C, Rio MC, Lerkowit N, Varro A, Giraud AS and Wang TC: Inhibition of gastric carcinogenesis by the hormone gastrin is mediated by suppression of TFF1 epigenetic silencing. Gastroenterology 140: 879-891, 2011.

19. Park WS, Oh RR, Park JY, Lee JH, Shin MS, Kim HS, Lee HK, Kim YS, Kim SY, Lee SH, Yoo NJ and Lee JY: Somatic mutations of the trefoil factor family 1 gene in gastric cancer. Gastroenterology 119: 691-698, 2000.

20. Sankpal NV, Mayo MW and Powell SM: Transcriptional repression of TFF1 in gastric epithelial cells by CCAAT/enhancer binding protein-beta. Biochim Biophys Acta 1728: 1-10, 2005.

21. Calnan DP, Westley BR, May FE, Floyd DN, Marchbank T and Playford RJ: The trefoil peptide TFF1 inhibits the growth of the human gastric adenocarcinoma cell line AGS. J Pathol 188: 312-317, 1999.

22. Lefebvre O, Chenard MP, Masson R, Linares J, Dierich A, LeMeur M, Wendling C, Tomasetto C, Chambon P and Rio MC: Gastric mucosa abnormalities and tumorigenesis in mice lacking the pS2 trefoil protein. Science 274: 259-262, 1996.

23. Feng Z, Chen J, Wei H, Gao P, Shi J, Zhang J and Zhao F: The risk factor of gallbladder cancer: hyperplasia of mucous epithelium caused by gallstones associates with p16/CyclinD1/CDK4 pathway. Exp Mol Pathol 91: 569-577, 2011.

24. Wang H, Liu H, Chen K, Xiao J, He K, Zhang J and Xiang G: SIRT1 promotes tumorigenesis of hepatocellular carcinoma through PI3K/PTEN/AKT signaling. Oncol Rep 28: 311-318, 2012.

25. Li L, Zhang J, Yang Y, Wang Q, Gao L, Yang Y, Chang T, Zhang X, Xiang G, Cao Y, Shi Z, Zhao M and Gao G: Single-wal carbon nanohorns inhibited activation of microglia induced by lipopolysaccharide through blocking of Sirt3. Nanoscale Res Lett 8: 100, 2013.

26. Li H, Bergeron L, Cryns V, Pasternack MS, Zhu H, Shi L, Greenberg A and Yuan J: Activation of caspase-2 in apoptosis. J Biol Chem 272: 21010-21017, 1997.

27. Fenoglio-Preisern CM, Wang J, Stemmermann GN and Noffsinger A: TP53 and gastric carcinoma: a review. Hum Mutat 21: 258-270, 2003.

28. Tahara E: Genetic pathways of two types of gastric cancer. IARC Sci Pub 157: 327-349, 2004.
29. Ballestar E and Wolffe AP: Methyl-CpG-binding proteins. Targeting specific gene repression. Eur J Biochem 268: 1-6, 2001

30. Babyatsky MW, deBeaumont M, Thim L and Podolsky DK: Oral trefoil peptides protect against ethanol and indomethacin-induced gastric injury in rats. Gastroenterology 110: 489-497, 1996.

31. Pera M, Heppell J, Poulsom R, Teixeira FV and Williams J: Ulcer associated cell lineage glands expressing trefoil peptide genes are induced by chronic ulceration in ileal pouch mucosa. Gut 48: 792-796, 2001

32. Newton JL, Allen A, Westley BR and May FE: The human trefoil peptide, TFF1, is present in different molecular forms that are intimately associated with mucus in normal stomach. Gut 46: 312-320, 2000

33. Herath NI, Leggett BA and MacDonald GA: Review of genetic and epigenetic alterations in hepatocarcinogenesis. $\mathrm{J}$ Gastroenterol Hepatol 21: 15-21, 2006.

34. Li HP, Leu YW and Chang YS: Epigenetic changes in virus-associated human cancers. Cell Res 15: 262-271, 2005.

35. Herceg Z: Epigenetics and cancer: towards an evaluation of the impact of environmental and dietary factors. Mutagenesis 22: 91-103, 2007.

36. Adrien LR, Schlecht NF, Kawachi N, Smith RV, Brandwein-Gensler M, Massimi A, Chen S, Prystowsky MB, Childs $\mathrm{G}$ and Belbin TJ: Classification of DNA methylation patterns in tumor cell genomes using a $\mathrm{CpG}$ island microarray. Cytogenet Genome Res 114: 16-23, 2006.

37. Lund $\mathrm{AH}$ and van Lohuizen M: Epigenetics and cancer. Genes Dev 18: 2315-2335, 2004

38. Sparmann A and van Lohuizen M: Polycomb silencers control cell fate, development and cancer. Nat Rev Cancer 6: 846-856, 2006.

39. Baylin SB: Mechanisms underlying epigenetically mediated gene silencing in cancer. Semin Cancer Biol 12: 331-337, 2002.

40. Hur K, Song SH, Lee HS, Ho Kim W, Bang YJ and Yang HK: Aberrant methylation of the specific $\mathrm{CpG}$ island portion regulates cyclooxygenase- 2 gene expression in human gastric carcinomas. Biochem Biophys Res Commun 310: 844-851, 2003.

41. Lee TL, Leung WK, Chan MW, Ng EK, Tong JH, Lo KW, Chung SC, Sung JJ and To KF: Detection of gene promoter hypermethylation in the tumor and serum of patients with gastric carcinoma. Clin Cancer Res 8: 1761-1766, 2002.

42. Carvalho B, Pinto M, Cirnes L, Oliveira C, Machado JC, Suriano G, Hamelin R, Carneiro F and Seruca R: Concurrent hypermethylation of gene promoters is associated with a MSH-H phenotype and diploidy in gastric carcinomas. Eur J Cancer 39: 1222-1227, 2003.

43. Byun DS, Lee MG, Chae KS, Ryu BG and Chi SG: Frequent epigenetic inactivation of RASSF1A by aberrant promoter hypermethylation in human gastric adenocarcinoma. Cancer Res 61: 7034-7038, 2001.

44. Sozzi G, Conte D, Leon M, Ciricione R, Roz L, Ratcliffe C, Roz E, Cirenei N, Bellomi M, Pelosi G, Pierotti MA and Pastorino U: Quantification of free circulating DNA as a diagnostic marker in lung cancer. J Clin Oncol 21: 3902-3908, 2003.

45. Toyota M and Issa JP: The role of DNA hypermethylation in human neoplasisa. Electrophoresis 21: 329-333, 2000.

46. Poulsen SS, Thulesen J, Christensen L, Nexo E and Thim L: Metabolism of oral trefoil factor 2 (TFF2) and the effect of oral and parenteral TFF2 on gastric and duodenal ulcer healing in the rat. Gut 45: 516-522, 1999.

47. Wong WM , Poulsom R and Wright NA: Trefoil peptides. Gut 44: 890-895, 1999.

48. Longman RJ, Douthwaite J,Sylvester PA, Poulsom R, Corfield AP, Thomas MG and Wright NA: Coordinated localisation of mucins and trefoil peptides in the ulcer associated cell lineage and the gastrointestinal mucosa. Gut 47: 792-800, 2000.

49. Dignass A, Lynch-Devaney K, Kindon H, Thim L and Podolsky DK: Trefoil peptides promote epithelial migration through a transforming growth factor beta-independent pathway. J Clin Invest 94: 376-383, 1994. 